\title{
Tausch und Geld: Ein Kommentar auf Grund von Georg Simmels Philosophie des Geldes
}

Citation for published version (APA):

Backhaus, J. G. (1996). Tausch und Geld: Ein Kommentar auf Grund von Georg Simmels Philosophie des Geldes. METEOR, Maastricht University School of Business and Economics. METEOR Research Memorandum No. 013 https://doi.org/10.26481/umamet.1996013

Document status and date:

Published: 01/01/1996

DOI:

10.26481/umamet.1996013

Document Version:

Publisher's PDF, also known as Version of record

\section{Please check the document version of this publication:}

- A submitted manuscript is the version of the article upon submission and before peer-review. There can be important differences between the submitted version and the official published version of record.

People interested in the research are advised to contact the author for the final version of the publication, or visit the DOI to the publisher's website.

- The final author version and the galley proof are versions of the publication after peer review.

- The final published version features the final layout of the paper including the volume, issue and page numbers.

Link to publication

\footnotetext{
General rights rights.

- You may freely distribute the URL identifying the publication in the public portal. please follow below link for the End User Agreement:

www.umlib.nl/taverne-license

Take down policy

If you believe that this document breaches copyright please contact us at:

repository@maastrichtuniversity.nl

providing details and we will investigate your claim.
}

Copyright and moral rights for the publications made accessible in the public portal are retained by the authors and/or other copyright owners and it is a condition of accessing publications that users recognise and abide by the legal requirements associated with these

- Users may download and print one copy of any publication from the public portal for the purpose of private study or research.

- You may not further distribute the material or use it for any profit-making activity or commercial gain

If the publication is distributed under the terms of Article $25 \mathrm{fa}$ of the Dutch Copyright Act, indicated by the "Taverne" license above, 


\title{
Tausch und Geld:
}

Ein Kommentar aufgrund von Georg Simmels Philosophie des Geldes

\author{
Prof. Dr. Jürgen G. Backhaus \\ Rijksuniversiteit Limburg \\ Vakgroep Algemene Economie \\ Postbus 616 \\ 6200 MD Maastricht \\ Nederland \\ tel: $31-43-3883652 / 3636$ \\ fax: $31-43-3258440$ \\ email: f.schijlen@algec.rulimburg.nl
}




\section{ABSTRACT}

\section{Tausch und Geld:}

\section{Ein Kommentar aufgrund vonGeorg Simmels Philosophie des Geldes}

Georg Simmel (1858-1918) veröffentlichte 1899 und1907 seine Philosophie des Geldes. ${ }^{1}$ Die

Rezeption in der amerikanischen Literatur aber aber auch in den Beitrag von Dietz betohnt den

Ökonomisch-Geldtheoretischen Aspekt dieses Beitrages und sucht ihn in der österreichischen Tradition der Volkswirtschaftslehre zu verankern. Aus der Sicht einer Theorie der Wirtschaftssysteme werden beide Einschätzungen dem Werke Simmels nicht gerecht.

Dieser kurze Beitrag hat drei Teile. Zunächst möchte ich das Werk aus der Sicht der Geschichte der ökonomischen Theorie einordnen und einen kurzen Überblick über seine Architektur geben.

Zweitens versuche ich einen Eindruck von den Fragestellungen zu geben, mit denen sich Simmel auseinandersetzte, sowie anzudeuten, wie er mit diesen Fragestellungen umging und zu welchen Ergebnissen er dabei kam.

Drittens versuche ich einen Zusammenhang zwischen Simmels Philosophie des Geldes und Fragen der wirtschaftlichen Systemtransformation herzustellen, um beispielhaft die aktuelle Bedeutung dieses Buches zu illustrieren.

J.E.L. codes: B3, E5, N2

1 Simmel, G. Fragment aus einer "Philosophie des Geldes". Jahrbuch für Gesetzgebung, Verwaltung und Volkswirtschaft. NF 23 (3), 1899, S. 813-854; und ders. Philosophie des Geldes. München und Leipzig, 1907. 


\section{Tausch und Geld:}

\section{Ein Kommentar aufgrund von Georg Simmels Philosophie des Geldes}

\section{Einleitung}

Raimund Dietz hat sich seit vielen Jahren mit der Marxistischen Wirttheorie und mit Simmels sogenannter Geldtheorie beschäftigt. ${ }^{2}$ Er hat diese Arbeiten über Fragen des Geldes als Institution nun soweit fortgeführt, daß er auch an den ordnungstheoretischen Vorstellungen anzuknüpfen sucht. Der eigentliche Zweck der Bemühung besteht darin, bestimmte Aspekte der neoklassischen Geldtheorie in Frage zu stellen.

Ob es sinnvoll ist, die denn doch wohl rudimentäre Geldtheorie bei Karl Marx über verschiedene andere Autoren gegen die moderne Neoklassik anzuführen, darüber müssen meines Erachtens Zweifel bestehen. Ich melde diese Zweifel sofort bei Beginn dieses Kommentars an um den Leser nicht in die Irre zu führen.

Die Brücke, die Raimund Dietz für sein Vorhaben gesucht hat, denn als Brücke dient ihm das Werk Simmels, ist an sich nicht schlecht gewählt. Simmel, mit Sombart einer der Begründer

2 Vgl. Dietz, R. (1976) "Sowjetökonomie: Warenwirtschaft oder Sachverwaltung. Ein Beitrag zur Begründung einer alternativen Theorie des Sozialismus". Studie zur Wirttheorie. Achberg; sowie ders. (1995), "Tausch, Geld und ökonomische Rationalität ein von Georg Simmel angeregter Beitrag zur Theorie der Wirtschaft". Manuskript, Wien. 
der deutschen Soziologie, arbeitete einen der Gedanken, die im Modernen Kapitalismus eine wesentliche Rolle spielen, philosophisch aus. Darauf muß die Betonung dann auch liegen, es handelt sich um eine philosophische Arbeit, Simmel ist in erster Linie nicht Ökonom, obwohl er mit Gewinn von Ökonomen gelesen werden kann. Darauf hinzuweisen ist nicht nur im Hinblick auf die verdienstvollen Arbeiten von Herrn Dietz wichtig, sondern auch im Hinblick auf die internationale Literatur.

Seit eine Übersetzung des Simmelschen Buches vorliegt ${ }^{3}$ haben Ökonomen verständlicherweise die wirtschaftlichen Aspekte der Simmelschen Philosophie des Geldes in den Vordergrund gerückt, wobei sie "wirtschaftlich" im Sinne dessen verstehen, was heute als wirtschaftlich im wissenschaftlichen Sinne betrachtet wird, z.B. jener Wissenskomplex, der mit der Kodierung des Journal of Economic Literature abgedeckt wird. Tatsächlich gehört aber Simmel in eine völlig andere Theorietradition, und er stand schon zur Zeit des Erscheinens seiner Theorie 1899 am Rande dessen, was damals zu den Wirtschaftswissenschaften gezählt werden konnte. Um den Kontext zu verstehen, muß man sich vor Augen halten, daß damals zu den Wirtschaftswissenschaften, die in der Regel wirtschaftliche Staatswissenschaften hießen, neben der Volkswirtschaftslehre und der Betriebswirtschaftslehre, die gerade entstand, auch die Soziologie, die politischen Wissenschaften, die Verwaltungswissenschaften, die moderne Wirtschaftsgeschichte sowie die Verwaltungsgeschichte, alle zu dem gehörten, was in demselben Kanon des Faches wirtschaftliche Staatswissenschaften gelehrt und geforscht wurde.

Zentral stand damals aber die Sozialpolitik, in deren Feld um neue Aufgaben und neue Orientierungen gerungen wurde. Dieses "Ringen” fand nach wissenschaftlichen Gesichtspunkten statt, zu denen in methodischer Hinsicht klärende Arbeiten in reicher Zahl entstanden, von

3 Simmel, G. The Philosphy of Money. Translation of the second enlarged edition by T. Bottomore and D. Frisbey. With an introduction by D. Frisbey. London and Boston. 1978. 
Menger über Schmoller, über Dilthey bis zu Weber und Sombart.

Ein anderes Mißverständnis, das nicht nur durch den vorliegenden Beitrag sondern auch durch die internationale Literatur scheinbar genährt wird, liegt in der Vorstellung, Simmels Beitrag sei der österreichischen Theorietradition zuzuordnen. Dietz verortet ihn zusammen mit Hayek, und Laidler und Rowe ${ }^{4}$ denken vor allem an Menger ${ }^{5}$.

Man muß sich vor Augen halten, wie sorgfaltig Laidler und Rowe formulieren. Keineswegs nehmen sie Simmel als Geldtheoretiker für sich in Anspruch. Sie machen schon in der Übersicht ihres Besprechungsaufsatzes jeden Leser darauf aufmerksam, daß sie einen winzigen Ausschnitt ins Auge fassen. Keineswegs behaupten Laidler und Rowe, Simmel habe österreichische (theoretische) Wurzeln. Sie vermuten dies, weisen dann aber auf den allgemeinen deutschen Theorieszusammenhang hin, der anders sei als der anglo-amerikanische. Einer der wesentlichen Unterschiede besteht darin, daß die deutsche Theorietradition wie geschildert einen integrativen Ansatz verfolgte, während die arbeitsteilige einzeldisziplinäre Forschung bereits früh seit Marshall in England bevorzugt wurde, und sich nach dem zweiten Weltkrieg auch in den Vereinigten Staaten von Amerika durchgesetzt habe. Einer der Vorreiter dieser vertiefenden Arbeitsverteilung war ausgerechnet Schumpeter, der in seinem eigenen Werk das Gegenteil verkörpert.

$4 \quad$ Laidler, D. und Rowe, N. (1980) "Georg Simmels Philosophy of Money; a Review Article for Economists". Journal of Economic Literature. 18, S. 97-105.

5 Laidler und Rowe schreiben: "Not only does Simmel seem to have drawn heavily on the work of Carl Menger, but later Austrian (not to mention Germans) in their turn drew on him. It is not just Simmel, but the entire German language tradition in monetary analysis that has been relatively neglected in contemporary monetary economics, both "Keynesian" and "Monetarist". The ideas to be found in the German language literature on money, not lest in Simmels philosophy, are in certain crucial respects different from those upon which most modern discussion is based, and we will therefore devote a considerable portion of this paper to the question of just what contemporary monetary economists do and do not have to learn from this literature." S. 97/98. The reference is to Carl Menger's Principles of Economics, 1971, translated by James Dingwall und Bert F. Hoselitz. Glencoe, Illinois, 1950. 
Dieser Hinweis ist für das Verständnis Simmels wichtig, und zwar nicht nur für den Dogmenhistoriker, dem diese Zusammenhänge in der Regel deutlich sind; sondern vor allem für den vergleichenden Wirtschaftssystemtheoretiker, der geneigt ist, seine Überlegungen vor allem von Eucken, Böhm und Hayek leiten zu lassen, die allesamt und ganz bewusst nur einen Ausschnitt dessen betrachten wollten, worauf es Simmel und seinem Lehrer Schmoller ankam. Denn hierin liegt der eigentliche Erkenntnisgewinn aus der Kontroverse mit Herrn Dietz. Wenn man Simmel in den Kontext zurückversetzt, in dem seine Arbeit tatsächlich entstanden ist, und dieser Kontext ist dokumentiert, dann kommt man im Hinblick auf Fragen des Wirtschaftssystemvergleichs und insbesondere der jetzt brennenden Fragen der Transformation von Wirtschaftssystemen auf andere Aspekte zu sprechen als jene, die Herr Dietz in den Vordergrund rückt. Diesen anderen Aspekten gilt dann auch dieser Kommentar.

Simmel war natürlich nicht daran interessiert, eine Brücke zwischen Marx und der modernen Neoklassik zu bauen. Er war auch nicht an einer Kritik der modernen Neoklassik interessiert. Vielmehr war er darin interessiert, an dem Forschungsvorhaben der Volkswirte jener Zeit unter Führung von Schmoller, Sombart, Spiethoff und anderer mitzuarbeiten und ein philosophisch-anthropologisches Fundament für die Frage zu liefern, wie der Geldverkehr einzuschätzen sei. Diese Frage ist in den letzten Jahren immer wieder zur Sprache gekommen, weil Wissenschaftler vor allem der Chicago Schule wie Becker, North, Stigler, Coase, und andere regelmäßig ihren Finger auf das Fehlversagen einer bestimmten öffentlichen Institution legen mit der dahinterliegenden Frage, wie es denn um das Verhalten der entsprechenden privaten Institution stehe. Auf diesem Gebiet liegt Simmels Feld und Bedeutung, und es ist einfach eindrucksvoll, wie er in seiner damals noch ungetümen deutschen Sprache die Grenzwertentscheidungen und ihre Folgen für das dauerhafte Verhalten von Individuen darstellt. Das Geld ist für ihn ein Merkpunkt, ein didaktisches Vehikel. Er hätte eine Fülle anderer 
Institutionen ohne weiteres wählen können, z.B. die Sprache.

Georg Simmel (1858-1918) veröffentlichte 1907 seine Philosophie des Geldes. ${ }^{6}$

Aus dem in der Fußnote mitgeteilten Zitat geht eindeutig hervor, daß es sowohl Schmoller als auch Simmel um das ging, was Sombart dann den "Geist" des Kapitalismus nannte, also das gesamte System der Wertorientierungen, das das Verhalten der wirtschaftenden Subjekte leitet, ohne notwendigerweise festgelegt zu sein, ohne notwendigerweise justiziabel zu sein, und doch wirksam und oft flexibel auf neue Umstände eingehend. ${ }^{7}$

Dieser rechtsökonomisch relevante Aspekt ist für die moderne Geldtheorie kaum von Bedeutung. Er ist aber von Bedeutung für die Fragen, die die Transformation von Wirtschaftssystemen vor allem in Europa heute dem Volkswirt stellen.

Dieser kurze Beitrag hat infolgedessen drei Ziele. Zunächst möchte ich das Werk aus der Sicht

6 München und Leipzig: Dunker \& Humblot. Für die Rezeption ist natürlich nicht nur der aussergewöhnliche Vorabdruck in dem von Schmoller herausgegebenen Jahrbuch interessant, sondern noch mehr der Besprechungsaufsatz, mit dem Gustav Schmoller selbst das dritte Heft des 25. Jahrgangs eröffnete. Er befaßte sich mit der ersten Auflage (Berlin und Leipzig, 1900) schilderte die Entstehungsgeschichte, machte einige kritische Anmerkungen, und legte vor allem Wert auf die sorgfältige wissenschaftliche Einordnung des Werkes.

Er schließt den Besprechungsaufsatz mit dem folgenden Zitat aus Simmel, in dem dieser sein eigenes Werk zusammenfasst: "Je mehr das Leben der Gesellschaft ein geldwirtschaftliches wird, desto wirksamer und deutlicher prägt sich in dem bewusten Leben der relativistische Charakter des Seins aus, da das Geld nichts anderes ist, als die in einem Sondergebilde verkörperte Relativität der wirtschaftlichen Gegenstände, die ihren Wert bedeutet."

Gustav Schmoller, "Simmels Philosophie des Geldes". Jahrbuch für Gesetzgebung, Verwaltung- und Volkswirtschaft im Deutschen Reich, 25.3, 1901, 799-816 (813).

7 Sowohl Simmel als auch Sombart haben ihre Arbeiten gründlich revidiert jeweils aufgrund der Kritiken Schmollers. Die zweite Auflage der Simmelschen Geldphilosophie wie auch die zweite Auflage des Sombartschen modernen Kapitalismus sind gerade auf diesem zentralen Punkt der wirtschaftlichen Bedeutung der normativen Orientierungen grundlegend neu bearbeitet worden. 
der Geschichte der ökonomischen Theorie einordnen und einen kurzen Überblick über seine Architektur geben.

Zweitens versuche ich einen Eindruck von den Fragestellungen zu geben, mit denen sich Simmel auseinandersetzte, sowie anzudeuten, wie er mit diesen Fragestellungen umging und zu welchen Ergebnissen er dabei kam.

Drittens versuche ich einen Zusammenhang zwischen Simmels Philosophie des Geldes und Fragen der wirtschaftlichen Systemtransformation herzustellen, um beispielhaft die aktuelle Bedeutung dieses Buches zu illustrieren.

I.

Georg Simmels Philosophie des Geldes besteht aus zwei großen Teilen, einem analytischen Teil und einem synthetischen Teil. Der analytische Teil besteht aus drei Kapiteln, die mit "Wert und Geld", "Der Substanzwert des Geldes" und "Das Geld in den Zweckreihen" überschrieben sind.

Simmel charakterisiert das Forschungsprogramm dieses analytischen Teils so: "Der erste Teil dieses Buches wird so das Geld aus denjenigen Bedingungen entwickeln, die sein Wesen und den Sinn seines Daseins tragen" (VI).

Für die Einordnung des Buches aus volkswirtschaftlicher Sicht ist zugleich als bemerkenswert festzuhalten, daß der Autor des "Das Wesen des Geldes" ${ }^{8}$ überschriebenen Bandes Simmel an keiner Stelle erwähnt. Dies ist nicht Ausdruck etwa der Unkenntnis oder einer oberflächlichen Vernachlässigung. Das Gegenteil ist der Fall, denn Simmel taucht in der History of Economic

$8 \quad$ Schumpeter, Joseph A. (1970). "Das Wesen des Geldes". 
Analysis $^{9}$ mindestens dreimal auf (S. 776, 785, 793), und sein Beitrag aus der Sicht der volkswirtschaftlichen Theorie des Geldes wird ausdrücklich gewürdigt.

In einem "From 1870 to 1914 and Later" überschriebenen Abschnitt äußert sich Schumpeter zunächst über das Ausbleiben großer philosophischer Würfe und dem Aufkommen von angewandten philosophischen Einzeluntersuchungen in dieser Periode.

Er schreibt auf S. 776: "The substitute for philosophy may take many forms and does not necessarily constitute philosophy as the universal science, scientia scientiarum, though the idea sometimes has been expressed in ways that suggest analogy with a holding company. Philosophy in this sense will look very different according to the individual philosopher's training. One type emerged from the hands of philosophers grounded in the physical sciences a type of positivism or monism that does not differ in any matter of principle from the "empirio-criticism" of Avenarius and Mach (IV). ${ }^{10}$ Another type which emerged from the hands of philosophers who were psychologists or sociologists by training later on came to be called Philosophical Anthropology (V) and is not always easy to distinguish from parts of Social Philosophy or straight Sociology. ${ }^{11}$

Both types invited misunderstandings of the specialist's theories ${ }^{12}$ and trespassed upon his

$9 \quad$ Schumpeter, Joseph A. (1954). "History of Economic Analysis".

10 Let us note the strong affinity of Mach's views with those of W.K. Clifford, K. Pearson, and J.H. Poincaré. Pearson's Grammar of Science (1892) and J.H. Poincarés La Valeur de la science (1904) are the two books I should recommend to readers desiring an easy guide to empirio-criticism.

11 Georg Simmel's Soziologie (1908) illustrates the latter point.

12 An amusing - or sad? - example of the occurrence of such misunderstandings even in the domain of physical sciences is the following: philosophers use the term Relativism frequently and in several different senses. It so happened that one of the most important novelties in the physics of the period was dubbed relativity theory, a term that has, of course, nothing whatever to do with historical or philosophical relativism in any sense. Nevertheless, a number of instances can be adduced of writers who made themselves ridiculous by interpreting the Einstein theory as a manifestation of the latter. I owe this fact (which at first I refused to believe) to Professor Philipp Frank. 
preserves, and they were not unnaturally resented ${ }^{13}$."

Fußnote 13 nun aber lautet: "Die Volkswirtschaftslehre wurde öfter das Opfer. Als Beispiel verweise ich auf Georg Simmels_Philosophie des Geldes". Diese Bemerkungen sind durchaus aufschlußreich, und sie sollten nicht vorschnell dahingehend interpretiert werden, Schumpeter habe sich mit dem Werk Simmels nicht sorgfältig auseinandergesetzt. Das Gegenteil ist der Fall.

Tatsächlich gibt Schumpeter Simmels Intentionen korrekt wieder, die allerdings wohl von Zeitgenossen falsch interpretiert wurden (und heute auch noch werden), so, als habe Simmel einen Beitrag zur volkswirtschaftlichen Theorie des Geldes leisten wollen, und die Bezeichnung als Philosophie diene lediglich der Immunisierung gegen die Fachkritik. ${ }^{14}$

Simmels Absichten decken sich aber keineswegs mit volkswirtschaftlichen Fragestellungen, denn er beschreibt das Programm des zweiten synthetischen Teiles wie folgt: "Die geschichtliche Erscheinung des Geldes, deren Idee und Struktur ich so aus den Wertgefühlen, der Praxis den Dingen gegenüber und den Gegenseitigkeitsverhältnissen der Menschen als ihren Voraussetzungen zu entfalten suche, verfolgt nun der zweite synthetische Teil in ihren Wirkungen auf

13 Economics, not sheltered like physics by age-old prestige, was frequently victimized. As an example, I mention G. Simmel's Philosophie des Geldes (1900), which treats of topics nearly all of which belong to the economist's sphere. Matters were not improved by Simmel's declaration that no proposition of the book was intended to be understood in the specialist's sense (ist einzelwissenschaftlich gemeint) - which was, of course, interpreted to mean that he would not accept criticism from the only people who understand, or should understand, the subject.

14 Gustav Schmoller weist in seiner Würdigung mehrfach daraufhin, er habe von den philosophischen Ansatz Simmels, den er selbst nur ungenügend aktiv beherrsche, sehr viel gelernt und dies auch in die entsprechenden Passagen seines Lehrbuches eingearbeitet. 
die innere Welt: auf das Lebensgefühl der Individuen, auf die Verkettung ihrer Schicksale, auf die allgemeine Kultur". (VI)

Keine volkswirtschaftliche Theorie hat derartige Fragestellungen, keine beschäftigt sich mit der inneren Welt und dem Lebensgefühl der Individuen oder der Verkettung ihrer Schicksale. Wir müssen Simmel ernst nehmen wenn er schreibt: "Keine Zeile dieser Untersuchungen ist nationalökonomisch gemeint". (VII)

Simmel erklärt zugleich, wie dieser apodiktische Satz zu verstehen ist.

Er schreibt: "Das will besagen, daß die Erscheinungen von Wertung und Kauf, von Tausch und Tauschmittel, von Produktionsformen und Vermögenswerten, die die Nationalökonomie von einem Standpunkte aus betrachtet, hier von einem anderen aus betrachtet werden.(...) So ist, daß zwei Menschen ihre Produkte gegeneinander vertauschen, keineswegs nur eine nationalökonomische Tatsache; denn eine solche, d.h. eine, deren Inhalt mit ihrem nationalökonomischen Bilde erschöpft wäre, gibt es überhaupt nicht. Jener Tausch vielmehr kann ganz ebenso legitim als eine psychologische, als eine sittengeschichtliche, ja als eine ästhetische Tatsache behandelt werden." (VII)

Übrigens ist Simmel der Ansicht, daß die Volkswirtschaftslehre die wirtschaftlichen Erscheinungsformen sehr viel gründlicher durchgearbeitet habe, als dies der Philosophie von ihren anderen Standpunkte aus gelingen könne. Insofern könne der Philosophie dann auch die Ergebnisse der Ökonomen zunächst als Tatsachen hinnehmen.Volkswirtschaftslehre und Philosophie sind einander komplementär. Der Fortschritt in der einen verdrängt die Bedeutung der anderen nicht.

Simmel sagt dies selbst so: "Ja, vielleicht würde selbst die vollendete Empirie die Philosophie als eine Deutung, Färbung und individuell auswählende Betonung des Wirklichen gerade so wenig ablösen, wie Vollendung der mechanischen Reproduktion der Erscheinungen, die bildende Kunst überflüssig machen würde."(V) Sowenig wie der Fortschritt in der Photog- 
raphie die bildende Kunst überflüssig macht, sowenig macht in Simmels Worten der Fortschritt in der empirischen Wirtschaftsforschung die Philosophie wirtschaftlicher Erscheinungen überflüssig.

\section{Damit handelt es sich bei Simmels Philosophie des Geldes aus der Sicht der volkswirt- schaftlichen Theorie des Geldes um ein aliud, ein anderes, nicht auf derselben Ebene vergleichbares. Es ist kategorisch unmöglich, etwa die neo-klassische Geldtheorie der Simmelschen Philosophie des Geldes gegenüberzustellen.}

Der Gegenstandsbereich der Wirtschaftswissenschaften verändert sich allerdings ständig, und die Wirtschaftswissenschaften greifen über wirtschaftliche Phänomene hinaus, sie machen auch andere Phänomene zu ihrem Gegenstand der Erklärung, insbesondere dann, wenn Wahlakte damit verknüpft sind.

In keinem Falle aber hat der ökonomische Imperialismus dazu geführt, daß das Lebensgefühl der Individuen und die Verkettung ihrer Schicksale zum Erklärungsgegenstand geworden wären.

Für die moderne Transaktionskostentheorie etwa kann man bei Simmel allenfalls Anregungen finden, aber keine Anleihen machen.

II.

Der zweite synthetische Teil der Simmelschen Philosophie des Geldes besteht wiederum aus drei Kapiteln, die mit "Die individuelle Freiheit", "Das Geldäquivalent personaler Werte" und "Der Stil des Lebens" überschrieben sind.

In dem letzten "Der Stil des Lebens" überschriebenen Kapitel finden sich eine Fülle von 
Beobachtungen, Erörterungen und Erwägungen, die für den Ökonomen anregend wirken, da über den Stilbegriff sich eine Brücke zwischen den Stil des Lebens und den Wirtschaftsstilen bietet, so daß den Betrachtungen auch eine wirtschaftliche Dimension zukommt. Jedoch ist es wichtig, sich stets vor Augen zu halten, was Simmel mit "Geld" meint, denn der Inhalt seines Begriffes ist wegen seines philosophischen Standpunktes mit jenem ökonomischer Geldbegriffe nicht nur nicht deckungsgleich, oft ist etwas ganz anderes (aliud) gemeint. Schön kommt dies heraus, wenn wir uns die Geldsubstitute vor Augen führen, an welche Simmel denkt, philosophisch und dies mit üblichen volkswirtschaftlichen Geldsubstituten vergleichen, heißt ihn verzeichnen. Simmel erörtert die Unpersönlichkeit des Austausches mit Geld, im Gegensatz zu anderen Austauschformen, und schreibt: "Was gegen Geld fortgegeben wird, gelangt an denjenigen, der das meiste dafür gibt, gleichgültig, was und wer er sonst sei; wo andere Äquivalente ins Spiel kommen, wo man um Ehre, um Dienstleistung, um Dankbarkeit sich eines Besitzes entäußert, sieht man sich die Beschaffenheit der Person an, der man gibt."

(S. 488-489)

Mit dieser Betonung der Geldsubstitute (Äquivalente) lenkt Simmel den Blick des Volkswirts auf Formen des Austausches, die normalerweise nicht Gegenstand seiner Betrachtung sind dies aber durchaus sein können. Tatsächlich gibt es inzwischen eine ökonomische Theorie des Geschenks ${ }^{15}$ und die Reputation (Ehre) wird in der volkswirtschaftlichen Organisationstheorie, insbesondere der Theorie der Unternehmung, im Zusammenhang mit den Bindungmöglichkeiten berücksichtigt.

Moderne Erscheinungen der Massenkommunikation, des Films, Fernsehens oder des Sports geben immer wieder Anlaß zu Kritik im Sinne einer allgemeinen Kulturkritik. Das Stichwort

15 Tullock, G. (1973), "The Charity of the Uncharitable", in: The Economics of Charity. 
"Cash Nexus" soll andeuten, daß Gefühle und Wertungen, aber auch künstlerische oder sportliche Ausdrucksformen irgendwie als Folge der Kommerzialisierung verflachen, synthetisch werden, oder andererseits ihren eigenen besonderen Reiz verlieren. Auf dieses Thema kommt auch Simmel zu sprechen, und wir finden die folgende eindrucksvolle Passage: "Die eigentümliche Abflachung des Gefühlslebens, die man der Jetztzeit gegenüber der einseitigen Stärke und Schroffheit früherer Epochen nachsagt; die Leichtigkeit intellektueller Verständigung, die selbst zwischen Menschen divergentester Natur und Position besteht - während selbst eine intellektuell so überragende und theoretisch so interessierte Persönlichkeit wie Dante noch sagt, gewissen theoretischen Gegnern dürfe man nicht mit Gründen, sondern nur mit dem Messer antworten; die Tendenz zur Versöhnlichkeit, aus der Gleichgültigkeit gegen die Grundfragen des Innenlebens quellend, die man zuhöchst als die nach dem Heil der Seele bezeichnen kann und die nicht durch den Verstand zu entscheiden sind - bis zu der Idee des Weltfriedens, die besonders in den liberalen Kreisen, den historischen Trägern des Intellektualismus und des Geldverkehrs gepflegt wird: alles dies entspringt als positive Folge jenem negativen Zuge der Charakterlosigkeit. An den Höhepunkten des Geldverkehrs wird diese Farblosigkeit sozusagen zur Farbe von Berufsinhalten". (S. 484)

Oft können wir feststellen, daß monetäre Entgeltung als Verfahren bei den Beteiligten oder in der Bevölkerung auf Unzufriedenheit stößt. Dies gilt insbesondere im Gesundheitswesen, läßt sich aber auch für den Bildungsbereich, den karitativen, den seelsorgerischen oder auch jenen Bereich zeigen, der traditionell besonders stark mit ehrenamtlichen Mitarbeitern arbeitet. Auch hier finden wir anschauliche Darstellungen bei Simmel, die wir beinahe nahtlos etwa in den heutigen Zusammenhang der Kostendämpfung im Gesundheitswesen übertragen können. Wenn der Krankenhausdirektor nüchtern auf Grund seiner Kosteninformationen abwägt und die Mittel entsprechend zuteilt, so stößt dies nicht nur bei Ärzten auf Unverständnis und wird oft als brutale Handlungsweise erfahren: "Gewiß hat die rein verstandesmäßige Behandlung 
der Menschen und Dinge etwas Grausames; aber sie hat dies nicht als positiven Impuls, sondern als einfache Unberührtheit ihrer bloß logischen Konsequenz durch Rücksichten, Gutmütigkeit, Zartheiten; weshalb denn auch entsprechend der rein geldmäßig interessierte Mensch es gar nicht zu begreifen pflegt, wenn man ihm Grausamkeit und Brutalität vorwirft, da er sich einer bloßen Folgerichtigkeit und reinen Sachlichkeit seines Verfahrens, ohne irgendeinen bösen Willen, bewußt ist." (S. 486)

III.

Diese drei beispielhaft ausgewählten Zitate aus dem sechsten Kapitel über den Stil des Lebens zeigen, daß Simmel uns vor allem Aufschluß über die Akzeptanz des Preissystems gibt, er uns also insbesondere darauf hinweist, welche Probleme sich ergeben können, wenn von einer Tauschform auf eine andere übergegangen wird, wenn also z.B. administrative Allokationsprozesse durch marktliche ersetzt werden. Dieses Problem stellt sich augenblicklich mannigfach bei der Transformation ehemals staatskapitalistischer Länder sozialistischer Prägung in Mittelund Osteuropa.

Simmels Perspektive ist nun nicht volkswirtschaftlicher Art, sein philosophischer d.h. ausdrücklich nicht marxistisch-leninistischer sondern hermeneutischer Standpunkt macht uns vielmehr auf solche Aspekte aufmerksam, die normalerweise außerhalb der Arbeit des Volkswirtes liegen, gleichwohl für die Umsetzung etwa eines Privatisierungsprogrammes von großer Bedeutung sein können.

Für den politischen Erfolg einer Privatisierungsstrategie kommt es ja nicht allein darauf an, ob geeignete Rechtsformen gefunden werden können, um die zu privatisierenden Einheiten funktionsgerecht aufzufangen; vielmehr kommt es darüberhinaus auch darauf an, wie die Menschen, die in und mit den neuen Organisationen umgehen müssen, diesen Übergang und 
die neuen Formen individuell erleben, wie also diese Politik sich auf das "individuelle Lebensgefühl" (Simmel) auswirkt.

Die Beispiele hierfür lassen sich in beliebiger Anzahl anführen, aber deren drei sollen hier genügen. Der Offizier der nationalen Volksarmee, der an seinen Eid gebunden seine Pflicht erfüllt, erfährt diesen Dienst und die damit verbundene Besoldung, die Ehrungen und den Respekt, der ihm gezollt wird, gänzlich anders als derselbe Mitarbeiter einer privaten Unternehmung zur Sicherung von Gebäuden und Transporten, selbst wenn jede einzelne seiner täglichen Handlungen gänzlich mit seinem früheren Dienst übereinstimmt. Umgekehrt fühlt sich der Mitarbeiter eines ehemals volkseigenen Betriebs, der auf eine Fülle von Sozialleistungen selbstverständlich zurïckgreifen konnte, wenn er sich politisch korrekt verhielt, in seinen Erwartungen enttäuscht, wenn die jährliche Urlaubsreise, der Gang zum Arzt oder die Sicherung eines Kindergartenplatzes jeweils erhebliche geldliche Aufwendungen erfordern, wo früher Wohlverhalten gegen diese Leistungen getauscht wurde.

Ebenso fühlen sich die Musiker etwa in einem international renommierten Orchester in ihrem Lebensgefühl betroffen, wenn die Auswahl der Stücke und der Programmplan plötzlich nach kommerziellen Gesichtspunkten getroffen werden müssen, wenn kulturelle Aspekte und das politische Wohlverhalten des Chefdirigenten früher den Durchschlag gegeben haben.

Diese den Stil des Lebens und damit auch des Wirtschaftens betreffenden Aspekte fallen normalerweise außerhalb der volkswirtschaftlichen Analyse und gehören in den Bereich der Wirtschaftssoziologie, nicht weil sie volkswirtschaftlich unbedeutend wären, sondern weil auf sie die volkswirtschaftlichen Methoden nicht überzeugend angewendet werden können. Zusammenfassend können wir feststellen, daß Simmels Philosophie des Geldes durchaus als Philosophie und nicht als volkswirtschaftlicher Beitrag intendiert war, und am besten auch so 
gelesen wird.

Die Volkswirtschaftslehre hat sich in den letzten 96 Jahren nicht so verändert, daß ein philosophischer Beitrag leichter integriert werden könnte als zur Zeit der ersten Publikation 1899. Zweitens muß diese Philosophie ausdrücklich im Kontext des Schmoller Programmes gesehen werden, der sich um eine umfassende sozial- und staatswissenschaftliche Theorie bemühte, der aber auch die Philosophie ausdrücklich ernst nahm aber nicht integrierte.

So wie er von Dilthey wesentliche Anregungen aufnahm, die dann zu dem "Methodenstreit" mit Menger führten, so nahm er auch Simmel ernst als Philosophen, ließ ihn wiederholt in seinem nur promovierten Gelehrten vorbehaltenen Seminar vortragen, und räumte seinen Schriften in seinem Publikationsorgan in jeder Hinsicht Vorrang ein, der auch von den Lesern nur so verstanden werden konnte. ${ }^{16}$

Die Bedeutung des Simmelschen Beitrages innerhalb der Schmollerschen Geldtheorie wird nicht nur aus den eigenen Bemerkungen Schmollers deutlich, sondern vor allem im Hinblick auf die heutige Lehre des Faches durch den allgemeinen Konsens, daß Schmoller überhaupt keine Geldtheorie gehabt habe. ${ }^{17}$

Aus der Sicht der Theorie der Wirtschaftssysteme ist es gewiß reizvoll, sich mit Simmels Philosophie des Geldes zu beschäftigen. Ob man aus derselben Sicht die neoklassische Geldtheorie angreifen möchte, steht auf einem anderen Blatt. Sie wurde für andere Anwendungen konzipiert.

16 Sowohl der Vorabdruck als auch die Rezension erschienen jeweils als "lead article".

17 Dies hängt damit zusammen, daß Schmoller seine Geldtheorie nicht geschlossen vorträgt. Die Grundlagen trägt er bei den "Massen" vor, die Einführung der Geldwirtschaft bei den der Entwicklung vorbehaltenen Kapiteln, und die Entartungen in dem eindrucksvollen Unterkapitel über die Hyperinflationen, das obwohl historisch aufgebaut geradezu prophetisch wirkt. 
Für die Wirtschaften im Transformationsprozeß, die augenblicklich das Hauptaugenmerk der Theoretiker der Wirtschaftssysteme verdienen, sind die anderen Aspekte m.E. wichtiger, die sich vor allem aus dem Kontext der Entstehung der Simmelschen Geldphilosophie erklären, die sich so auch als eine Philosophie erweist, und nicht als eine angewandte Theorie der Wirtschaftswissenschaften.

Prof. Dr. Jürgen G. Backhaus

\section{Literatur}

Clifford, W.K., Pearson, K., Poincaré, J.H. (1892), "Pearson's Grammar of Science” (1892)

Poincarés, J.H. (1904), La Valeur de la science (1904)

Dietz, R. (1976) “Sovietökonomie: Wahrenwirtschaft oder Sachverwaltung. Ein Beitrag zur Begründung einer alternativen Theorie des Sozialismus" in: Studie zur Wirttheorie. Achberg, 1976.

Dietz, R. (1995), “Tausch, Geld und ökonomische Rationalität - ein von Georg Simmel angeregter Beitrag zur Theorie der Wirtschaft" in: Manuskript. Wien, 1995.

Laidler, D., Rowe, N. (1980) "Georg Simmels Philosophy of Money; a Review Article for Economists". Journal of Economic Literature. 18, S. 97-105. 
Laidler, D., Rowe P. 97/98. Carl Menger's Principles of Economics (1971), translated by Dingwall, J., Hoselitz, B.F. Glencoe, Illinois, 1950.

Schmoller, G. (1901), "Simmels Philosophie des Geldes”. Jahrbuch für Gesetzgebung, Verwaltung- und Volkswirtschaft im Deutschen Reich, 25.3, 1901, 799-816 (813).

Schumpeter, J.A. (1954), History of Economic Analysis. New York, 1954.

Schumpeter, J.A. (1970), Das Wesen des Geldes. Göttingen,1970.

Simmel, G. Philosophie des Geldes, München und Leipzig, 1907.

Simmel, G. The Philosphy of Money. Translation of the second enlarged edition by Bottomore, T., Frisbey, D. With an introduction by D. Frisbey. London and Boston. 1978.

Tullock, G. "The Charity of the Uncharitable", in: The Economics of Charity. London, 1973. 